\title{
Unravelling the electronic nature of the radiative cooling of cobalt clusters
}

\author{
Kristien Peeters $\odot,{ }^{1}$ Ewald Janssens $\odot,{ }^{1}$ Klavs Hansen $\odot,{ }^{1,2}$ Peter Lievens $\odot,{ }^{1}$ and Piero Ferrari $\odot{ }^{1, *}$ \\ ${ }^{1}$ Quantum Solid-State Physics, Department of Physics and Astronomy, KU Leuven, Celestijnenlaan 200d box 2414, 3001 Leuven, Belgium \\ ${ }^{2}$ Center for Joint Quantum Studies and Department of Physics, School of Science, Tianjin University, 92 Weijin Road, Tianjin 300072, China
}

(Received 2 May 2021; accepted 17 August 2021; published 8 September 2021)

\begin{abstract}
In this paper, we report on the experimental determination of photon emission rates of laser-excited cobalt clusters, $\mathrm{Co}_{n}{ }^{+}(n=5-23)$, deduced from fragmentation mass spectrometry and metastable decay fractions. The rates are so high that they can only be ascribed to recurrent fluorescence (RF), a process where emitting states are populated by inverse internal conversion, followed by photon emission. Cooling via electronic states is confirmed by quantitative agreement with calculated rates using the low-lying electronic transitions predicted by timedependent density functional theory calculations for $n=5-10$, which are performed considering all electrons and including relativistic effects implicitly. The outstanding agreement between experiment and theory provides clear evidence that the clusters radiate via electronic states, being a consistent theoretical and experimental study invoking RF.
\end{abstract}

DOI: 10.1103/PhysRevResearch.3.033225

\section{INTRODUCTION}

The emission of photons by isolated and thermally equilibrated clusters can occur via two different internal transitions: vibrational cooling (VC) and recurrent fluorescence (RF). $\mathrm{VC}$ involves the emission of relatively low-energy photons in vibrational transitions with low oscillator strengths and therefore occurs on the millisecond or longer time scale [1,2]. $\mathrm{RF}$, in contrast, occurs at time scales that can be as short as microseconds. Such a fast stabilization of a hot particle has direct consequences in diverse fields, including the size-selected production of nanoparticles, catalysis, and astrophysics [3]. To date, however, there is no widespread agreement that RF is responsible for this fast cooling because predictions from quantum chemical calculations on many occasions have disagreed with measured cooling rates.

RF processes occur because excited electronic states can be populated thermally via inverse internal conversion (IIC), in which vibrational energy is converted into electronic energy, followed by photon emission from such states if the transitions are optically allowed [4,5]. Fast radiative cooling, consistent with RF, has been observed from size-selected carbon-based molecules and metal clusters [6-9], as well as from broad mass distributions of refractory metal particles [10-12]. Moreover, visible photons emitted by small carbonbased molecules have been detected in ion storage devices $[13,14]$. Despite all the experimental evidence in favor of RF, theoretical calculations assuming this process did not support

\footnotetext{
*piero.ferrari@kuleuven.be

Published by the American Physical Society under the terms of the Creative Commons Attribution 4.0 International license. Further distribution of this work must maintain attribution to the author(s) and the published article's title, journal citation, and DOI.
}

the experimental data $[6,15]$, leaving room for skepticism. In $\mathrm{Au}_{n}^{+}$clusters, for example, fast radiative cooling has been observed for several sizes, whereas quantum chemical calculations of electronic excitations yield states too high in energy to explain the experimental results [6]. Only very recently have infrared spectroscopy experiments detected what seems to be a low-lying electronic excitation in $\mathrm{Au}_{10}^{+}$[16], supporting the predictions made in Ref. [6], based purely on experimental evidence.

In this paper, we focus our attention on the radiative cooling of cationic cobalt clusters. Electron correlations in small cobalt clusters have attracted significant attention in recent years. X-ray magnetic circular dichroism spectroscopy measurements have revealed large spin and orbital magnetic moments for $\mathrm{Co}_{n}^{+}(n=4-15)$ clusters [17,18]. Cunningham et al. [19] recently demonstrated the involvement of lowenergy excited states in the $\mathrm{NO}_{2}$ binding to small $\mathrm{Co}_{n}^{+}(n \leqslant 5)$ clusters, and experiments with a free-electron laser have shown the population of electronic states after vibrational excitation of neutral $\mathrm{Co}_{n}(n=9,10,13)$ clusters [20]. These results all depend on the high density of states near the highest occupied molecular orbital (HOMO), due to the open $3 d$-shell electronic configuration and energetic competition between $3 d$ and $4 s$ occupancy. This electronic structure meets the criteria for expecting high rates of radiation. Moreover, the geometries of $\mathrm{Co}_{n}^{+}$clusters have been determined in the $n \leqslant 8$ size range by a combination of infrared spectroscopy experiments and density functional theory (DFT) calculations [21]. This unique combination makes cobalt clusters an excellent testing ground for the presence and magnitudes of thermally excited electronic states in few-body systems.

Here, we report a mass spectrometric and computational analysis of the radiative cooling of laser-excited cationic cobalt clusters $\mathrm{Co}_{n}^{+}(n=5-23)$. The experimental emission rates are so high that they can only be explained by RF. Notably, the experimental radiation rates are in quantitative 


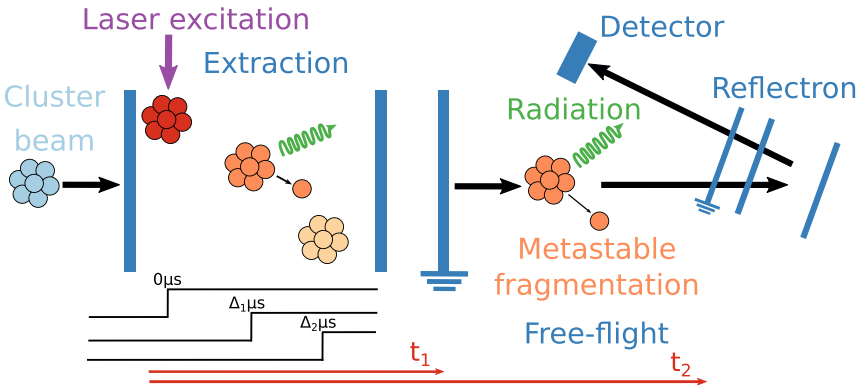

FIG. 1. Scheme of the mass spectrometer (not to scale), detailing the laser excitation process and the time delay $(\Delta t)$ between excitation and extraction.

agreement with those calculated for $n=5-10$, using the low-lying electronic transitions predicted by time-dependent DFT (TD-DFT). Therefore, our analysis provides a clear reconciliation of the theoretical understanding of RF with the experimental observations.

\section{METHODS}

\section{A. Experimental}

The cobalt clusters were produced by laser ablation and analyzed by reflectron time-of-flight mass spectrometry in a setup described in detail in Ref. [22]. The cluster source was kept at room temperature, with $\mathrm{He}$ used as the carrier gas. Clusters initially produced as ions were electrostatically deflected from the molecular beam, while those produced as neutrals in the source were exposed to the focused ultraviolet (UV) laser light of a nanosecond ArF excimer laser (193 nm, $5 \mathrm{~mJ}$ per pulse). Under these high-fluence conditions, multiphoton absorption takes place, inducing a combination of fast ionization and fragmentation of the clusters, as the electronic energy is rapidly converted into vibrations [23]. Photofragmentation has been used on many occasions for investigating cluster relative stabilities [24-28], and the principles of the present experiments do not differ from those.

The radiative cooling was monitored by its influence on the metastable decay fraction $\left(M_{n}\right)$, defined as the relative amount of the clusters that fragment during free flight (after leaving the extraction and before entering the reflectron) in the mass spectrometer (within microsecond time scales), with respect to those that fragment practically instantaneously upon laser excitation (prompt fragments). The technique is illustrated in Fig. 1, showing the mass spectrometer schematically. By detuning the reflectron fields from the optimal resolution values, the clusters that fragment during free flight between the initial acceleration and the entry into the reflectron can be positioned aside the product cluster in the time-of-flight spectrum. Metastable fragmentation occurs within a well-defined time window, between the moment the cluster leaves the extraction stage of the mass spectrometer $\left(t_{1}\right)$ and the time the cluster reaches the reflectron $\left(t_{2}\right)$. The geometry defining these times is shown in Fig. 1 by red arrows and is calculated by using the known dimensions of the mass spectrometer and the voltages applied to acceleration and reflectron electrodes. As during fragmentation the velocity of the fragment is unchanged $[29,30]$, metastable fragments are seen as sharp peaks in mass spectra, independent of the precise position in the free-flight region the fragmentation occurs [31]. Further details of the experimental approach can be found elsewhere [31,32]. For all investigated clusters, monomer loss is observed as the dominating metastable channel, as can be identified by comparing the time separation between prompt and metastable fragments in mass spectra [31].

The analysis of the number of metastable fragments is based on the fact that the internal energy of a metastable cluster has an upper and a lower limit [26]. If too high, fragmentation will occur very fast, hence when the cluster is still at the acceleration stage of the mass spectrometer. In contrast, if too low, fragmentation will take place after the cluster leaves the free flight region. Importantly, this window of internal energies for the metastable fragments depends on a combination of cluster-specific parameters [33] and the settings of the mass spectrometer but is independent on the cluster temperature before the laser excitation.

\section{B. Computational}

DFT calculations were performed for $\mathrm{Co}_{n}^{+}$clusters in the $n \leqslant 10$ size range, at a high level of theory. The geometries of the clusters in the $n=5-8$ range are known from infrared multiple photon dissociation spectroscopy experiments [21]. In contrast, the putative lowest-energy structures of $\mathrm{Co}_{9}^{+}$and $\mathrm{Co}_{10}^{+}$have been found only by a search based on DFT energies [34]. Here, we adopt the geometries determined in those studies. The calculations were performed using the ORCA 4.1.2 software package [35], employing the Perdew-Burke-Ernzerhof functional in conjunction with the large Def2-TZVPP basis set. Benchmark studies have shown that generalized gradient approximation functionals are the most appropriate to describe first-row transition metal systems [36]. All electrons were considered for the calculations. Moreover, the D3BJ correction was applied to include dispersion forces, and relativistic effects were accounted implicitly by using the zeroth order regular approximation (ZORA). The applied level of theory was successfully used for the geometry determination of neutral $\mathrm{Co}_{n}(n=4-10,13)$ clusters [37]. Harmonic vibrational frequencies were calculated to corroborate that the geometry optimizations converged into true minima on the potential energy surface of the clusters.

\section{RESULTS AND DISCUSSIONS}

A typical mass spectrum of $\mathrm{Co}_{n}^{+}(n=5-23)$ clusters after photoexcitation is presented in Fig. 2(a). The metastable fragments are seen as the secondary peaks in the mass spectrum, marked by asterisks, whereas the clusters fragmenting immediately after laser excitation (prompt) are the main peaks, of which a few sizes are labeled. There is a distinct size-dependence of the recorded metastable fractions, as is presented in Fig. 2(b). Moreover, Fig. 2(c) [zoom of panel (a) in the 630-890 u mass range] depicts as an example the fragmentation channel of $n=14$.

To extract (size-dependent) rates of radiative cooling, a small and variable time delay is added between the creation of the hot cationic clusters by the UV laser excitation and the time the clusters are extracted into the mass 

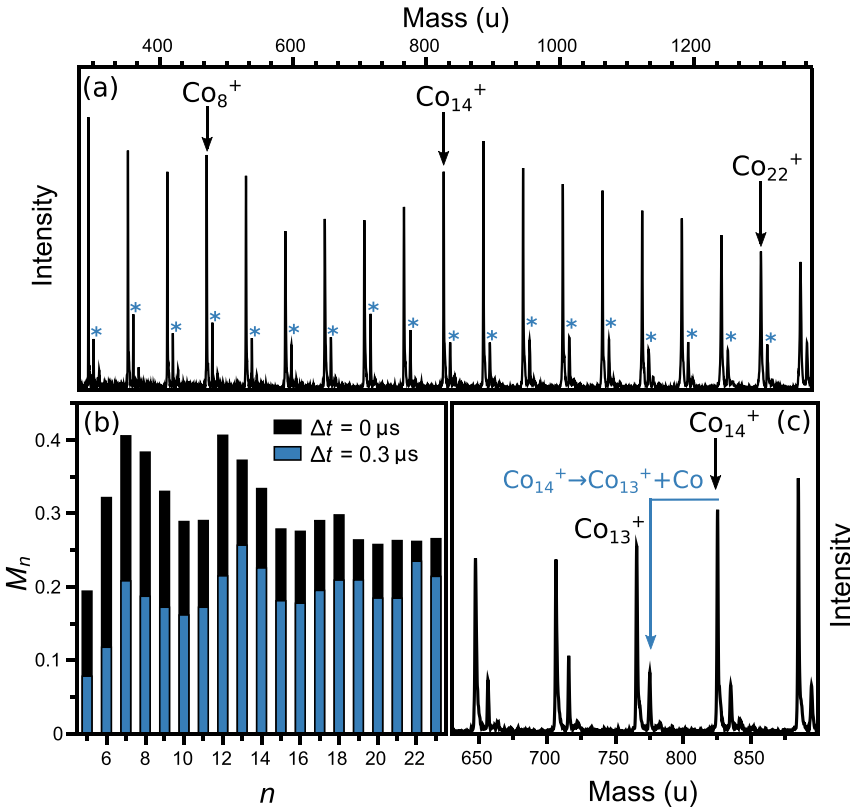

FIG. 2. (a) Representative mass spectrum of photofragmented $\mathrm{Co}_{n}^{+}$clusters, recorded at $\Delta t=0 \mu \mathrm{s}$. The metastable species are marked by an asterisk. The small additional peaks are oxygen contaminations. (b) Metastable fractions as a function of cluster size, recorded with prompt extraction $(\Delta t=0 \mu \mathrm{s}$; black $)$ and $\Delta t=0.3 \mu \mathrm{s}$ (blue). (c) Zoom of the 630-890 u mass range of the spectrum in panel (a), where the fragmentation channel $\mathrm{Co}_{14}^{+} \rightarrow \mathrm{Co}_{13}^{+} \rightarrow \mathrm{Co}$ is depicted. The most intense peaks are the clusters that are detected with the same mass as they are accelerated.

spectrometer. Such a time delay $(\Delta t)$ acts as a cooling time during which the highly excited clusters can fragment and radiate before they are analyzed mass spectrometrically. Measured metastable fractions recorded at two time delays are depicted in Fig. 2(b). The method is based on the fact that, under the high-fluence conditions of the laser excitation, the clusters are excited to a broad internal energy distribution, both because of Poisson statistics and variations of the laser fluence across the beam [38]. For clusters solely cooling down via fragmentation, such excitation conditions induce decays following a $1 / t$ time dependence [39]. If radiation also occurs in parallel with fragmentation, the $1 / t$ time dependence is quenched, and the expression for $M_{n}$ becomes

$$
M_{n}=C_{n} \int_{t_{1}+\Delta t}^{t_{2}+\Delta t} \frac{e^{-k_{p} t}}{t} d t,
$$

with $k_{p}$ the photon emission rate constant and $C_{n}$ a time independent constant. Equation (1) is valid for radiation of either electronic or vibrational nature if the emission of a single photon quenches any further fragmentation or in the absence of radiation, for which $k_{p}=0$ [3]. Here, $k_{p}$ is then determined by measuring $M_{n}$ for different $\Delta t$ values and fitting Eq. (1) to these data. This procedure has been applied successfully to determine radiation rates of diverse metal clusters [3].

Figure 3(a) presents examples of the procedure for the photoexcited clusters $\mathrm{Co}_{5}^{+}$and $\mathrm{Co}_{14}^{+}$. This figure shows a fitting to the experimental data using Eq. (1) as well as the time-dependence of Eq. (1) with $k_{p}=0$. Clearly, the latter
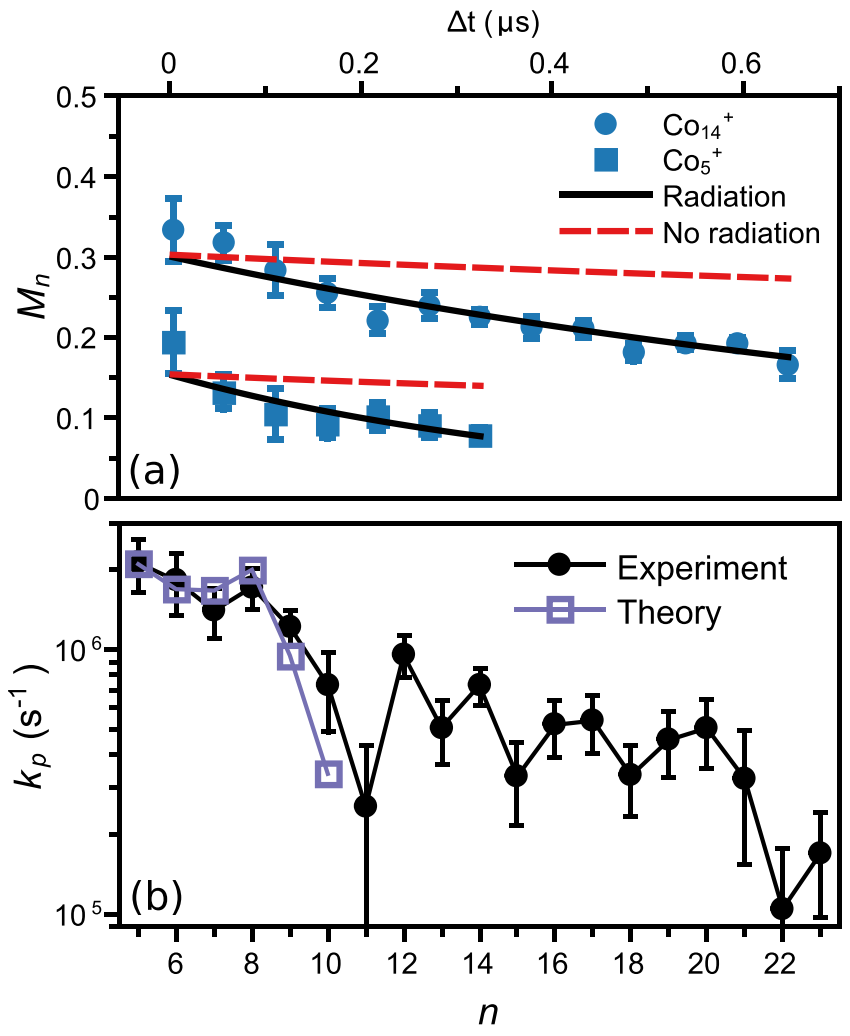

FIG. 3. (a) Metastable fractions as function of delayed time of extraction for $\mathrm{Co}_{5}^{+}$and $\mathrm{Co}_{14}^{+}$. The experimental points are fitted by Eq. (1), accounting for the presence of radiative cooling (continuous line). In addition, the delay dependence accounting for fragmentation as the only energy dissipation mechanism is shown (dashed line). (b) Experimental (full circles, $n=5-23$ ) and calculated (open squares, $n=5-10) \mathrm{Co}_{n}^{+}$rates of radiation.

cannot capture the $\Delta t$ dependence of $M_{n}$, whereas the former provides a good fit, consistent with radiation competing with fragmentation as a cooling channel. The obtained radiative rate constants, given in Fig. 3(b) (filled circles), are very high, for $n \leqslant 9$, with the photon emission rate constant $k_{p}=$ $2.1 \times 10^{6} \mathrm{~s}^{-1}$ for $\mathrm{Co}_{5}^{+}$, the highest value reported to date for thermal radiation. The rates show some size-to-size variations in addition to a general decrease with size, like previous observations [3].

The RF rate $\left(k_{\mathrm{RF}}\right)$ of a cluster can be expressed by Eq. (2), with $A$ the spontaneous decay Einstein coefficient of the electronic transition from a state of energy $E_{i}$, and $\rho_{n}$ the level density of the $n$-atom cluster [39]:

$$
k_{\mathrm{RF}}=A \frac{\rho_{n}\left(E-E_{i}\right)}{\rho_{n}(E)\left[1-\frac{\rho_{n}\left(E-2 E_{i}\right)}{\rho_{n}\left(E-E_{i}\right)}\right]} .
$$

The photon emission rate constant $k_{\mathrm{RF}}$ is thus the product of $A$ and the population determined by the level density, as in any other activated process, and an additional term in the denominator (within square brackets) accounting for stimulated emission. This expression, including the appearance of stimulated emission, is obtained by performing a detailed balance calculation [39]. The difference in time scales of VC and $\mathrm{RF}$ radiation arises because the Einstein $A$ coefficients of electronic transitions are orders of magnitude larger than 
(a)

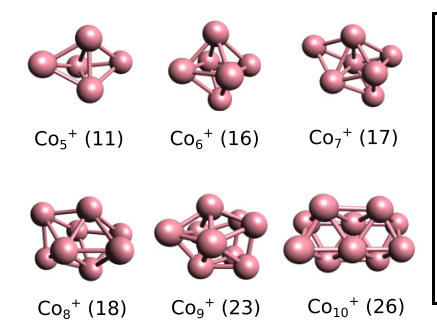

(c)

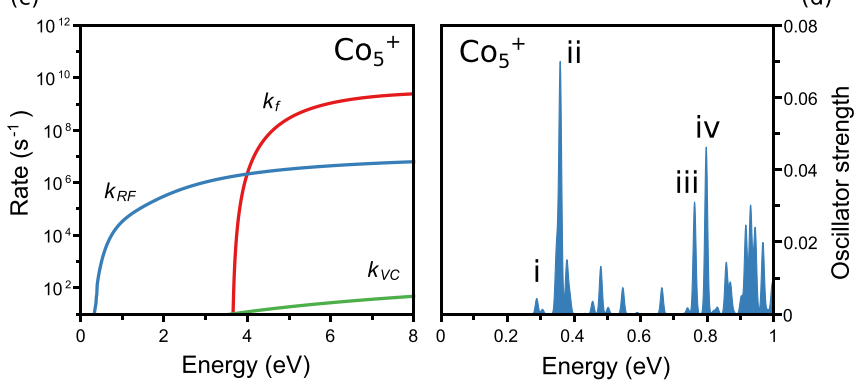

FIG. 4. (a) Geometries of $\mathrm{Co}_{n}^{+}(n=5-10)$ clusters. The adopted multiplicities are shown in parenthesis. (b) Dissociation energies calculated for the monomer and dimer loss channels. (c) Decay rates calculated for the $\mathrm{Co}_{5}^{+}$cluster. The different curves correspond to fragmentation $\left(k_{f}\right)$, recurrent fluorescence $\left(k_{\mathrm{RF}}\right)$, and vibrational cooling $\left(k_{\mathrm{VC}}\right)$. (d) Oscillator strength of electronic excitations as a function of the excitation energy of $\mathrm{Co}_{5}^{+}$up to $1 \mathrm{eV}$. An artificial linewidth of $0.01 \mathrm{eV}$ was used for the plot. Four transitions are labeled, as detailed in Fig. 5.

vibrational ones. This big enhancement in emission rate is only partly offset by the small population of the emitting state. Whereas excited state populations can be seriously reduced by the ratio of level densities in Eq. (2), which is effectively a Boltzmann factor [6], the ability to have enough thermally excited electrons is nevertheless well established. For example, this was seen in the mentioned population of electronic states via vibrational excitation of $\mathrm{Co}_{n}(n=9,10,13)$ [20] and for $\mathrm{Nb}_{3} \mathrm{C}_{2}$ and $\mathrm{Nb}_{6} \mathrm{C}_{5}$ [40].

The likelihood of IIC and hence photon emission strongly decreases with increasing energy of the involved excited state [6]. Therefore, a fundamental requirement for RF to occur is the existence of low-lying and optically active electronically excited states in the cluster. Such a low-lying electronic mode was recently detected for the $\mathrm{Ta}_{5} \mathrm{C}_{3}$ cluster in infrared spectroscopy experiments [41] as well as very recently also for $\mathrm{Au}_{10}^{+}$[16].

The converged $\mathrm{Co}_{n}^{+}$structures are depicted in Fig. 4(a), while XYZ coordinates are listed in the Supplemental Material (SM) [42]. The spin states proposed in Ref. [21] were assumed for the $n=5-8$ size range. These spin states differ slightly (except for $\mathrm{Co}_{6}^{+}$) from those quantified on x-ray magnetic circular dichroism spectroscopy experiments [17]. In the SM [42], an alternative result for $\mathrm{Co}_{5}^{+}$, based on the spin state determined in Ref. [17] is presented. In both cases, low-lying electronic excited states are predicted, which gives rise to similar radiation rates. Furthermore, for $\mathrm{Co}_{9}^{+}$and $\mathrm{Co}_{10}^{+}$, the spin states experimentally determined in Ref. [18] were assumed.
Based on the geometries shown in Fig. 4(a), dissociation energies $\left(D_{n}\right)$ are calculated as $D_{n}=E\left(\mathrm{Co}_{n-m}^{+}\right)+E\left(\mathrm{Co}_{m}\right)-$ $E\left(\mathrm{Co}_{n}^{+}\right)$, with $E$ the ground state energy of the cluster in parenthesis, and $m=1$ or 2 , corresponding to the neutral monomer or dimer loss channel. The calculated energies are shown in Fig. 4(b). The monomer loss channel is energetically favorable for all cases, although a complete analysis of the branching ratios, which is outside the scope of this paper, would require consideration of the entropy associated with rotational degrees of freedom. The trend agrees with the dissociation energies found in Ref. [43], although our values are higher. The vibrational frequencies of the clusters were used, together with the computed $D_{n}$, to obtain the fragmentation rate constants $\left(k_{f}\right)$ as a function of internal energy. For this purpose, level densities were computed using the BeyerSwinehart algorithm [39]. As an example, $k_{f}$ is plotted as a function of internal energy for $\mathrm{Co}_{5}^{+}$[red line in Fig. 4(c)]. In addition, using the calculated vibrational modes of $\mathrm{Co}_{5}^{+}$, also the $\mathrm{VC}$ rate constants $\left(k_{\mathrm{VC}}\right)$ were computed [green line in Fig. 4(c)].

For each excitation energy, the decay channel with the highest rate in Fig. 4(c) dominates. For all excitation energies, $k_{\mathrm{VC}} \ll k_{f}$ and is not significant on the experimentally relevant time scale of microseconds, demonstrating that $\mathrm{VC}$ is not responsible for the quenching of the metastable fragmentation. At most, $k_{\mathrm{VC}}$ reaches values $\sim 50 \mathrm{~s}^{-1}$, corresponding to a cooling time of $20 \mathrm{~ms}$. The $k_{\mathrm{RF}}$ (blue line) values, in contrast, are signicantly higher and allow an observable crossover from predominantly fragmentation to predominantly radiative cooling.

Given the broad energy distributions of the excited clusters, it is not possible to assign a single energy to the clusters. Nevertheless, the clusters that decay at a specific time are the clusters that have decay constants corresponding to this time. When both unimolecular decays and photon emission occur at the same time, they must therefore have similar rate constants. This is precisely the situation when metastable unimolecular decay is suppressed by radiative cooling, a point where both processes compete. Hence, the theoretical prediction for the experimentally measured value of $k_{p}$ in these experiments is the point where the curves for $k_{f}$ and $k_{\mathrm{RF}}$ cross [39].

The RF rate constants shown in Fig. 4(c) were calculated by combining the TD-DFT computations and statistical modeling. The TD-DFT results provide the energies and oscillator strengths of the dipole-allowed electronic transitions between excited states and the ground state, which are then used to calculate the $A$ coefficient associated with that transition, as $A=7.421 \times 10^{-22} \mathrm{~Hz}^{-1} f v_{0}^{2}$ (with $f$ the oscillator strength and $h v_{0}$ the transition energy) [6]. For each optically active state, a rate constant was calculated, using Eq. (2), and the total rate was obtained as the sum over all transitions. No further parameters are used in the calculation. The computed electronic transitions of $\mathrm{Co}_{5}^{+}$, up to an energy of $1 \mathrm{eV}$, are shown in Fig. 4(d). In the SM [42], the spectrum including the visible range up to $4 \mathrm{eV}$ is given, as are the low-energy spectra up to $1 \mathrm{eV}$ for the $\mathrm{Co}_{n}^{+}(n=6-10)$ clusters. The TD-DFT calculations predict several electronic transitions $<1 \mathrm{eV}$ with significant oscillator strength. The existence of those lowenergy excitations explains the high $k_{p}$ rate of $\mathrm{Co}_{5}^{+}$. Similarly, 
(a)

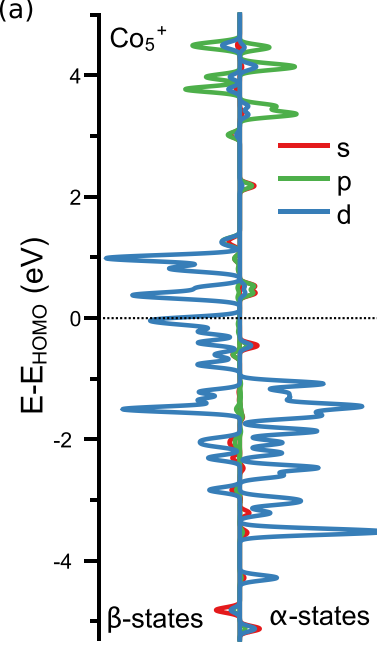

(b)

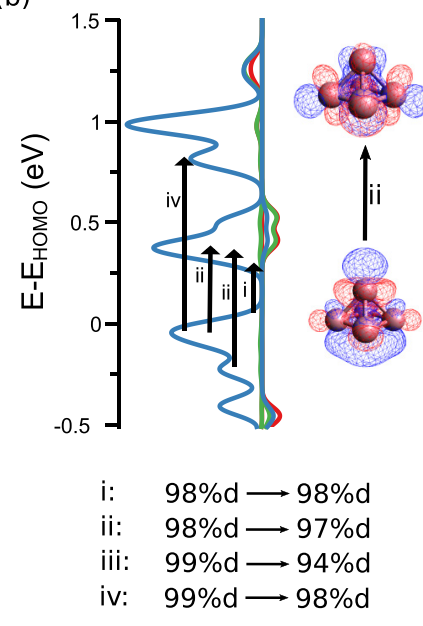

FIG. 5. (a) Density of states of the $\mathrm{Co}_{5}^{+}$cluster, projected into states of $s, p$, and $d$ atomic character. Energies are presented with respect to the highest occupied molecular orbital (HOMO) state (marked by a dotted line). $\alpha$ and $\beta$ states appear on the right and the left side of the plot, respectively. (b) Zoom in of the partial density of states (PDOS) in the region around the HOMO state, highlighting by arrows the electronic transitions labeled in Fig. 3(d). On the right side, the molecular orbitals involved in transition (ii) are shown. The contributions of atomic $d$ character in the (i)-(iv) electronic transitions of $\mathrm{Co}_{5}^{+}$are mentioned in the bottom inset.

very high photon emission rate constants are found for the other cluster sizes.

The calculated values are shown in Fig. 3(b) (open squares), which are found to be in good agreement with the experimental results. This is particularly the case for $n=6-8$, for which the cluster geometries are known experimentally. A slightly larger deviation from the experimental value is seen for $n=10$ (as a reminder, the geometries of the $n=9$ and 10 clusters have only been determined by DFT). Nevertheless, the predicted radiation rate of $\mathrm{Co}_{10}^{+}$lies within $1 \sigma$ of the experimental value, and the decrease of $k_{p}$ is nicely reproduced by the calculations.

The possibility that more than one isomeric geometry is present in the cluster beam and therefore contributes to the measured radiation rate is explored for $\mathrm{Co}_{5}^{+}$, for which two additional low-lying isomers are considered, assuming the relative weights of the isomers in a vibrational harmonic approximation and in the high temperature limit [39]. Details of the calculation are found in the SM [42]. Given the small relative population of the additional isomers, the calculated radiation rate $\left(1.99 \times 10^{6} \mathrm{~s}^{-1}\right)$ is like that in Fig. 3(b) $\left(2.10 \times 10^{6} \mathrm{~s}^{-1}\right)$

The electronic transitions computed for $\mathrm{Co}_{5}^{+}$are further analyzed in Fig. 5. In panel (a), the density of states of the cluster is projected into states with atomic $s, p$, and $d$ character

[partial density of states (PDOS)]. Many states of mainly $d$ character and spin down $(\beta)$ are located around the HOMO (zero energy), while spin-up $(\alpha)$ states are found at lower energies, consistent with the high multiplicity of the cluster $(2 S+1=11)$. The four electronic transitions labeled in Fig. 4(d) are shown by arrows in Fig. 5(b) [zoom of the PDOS in Fig. 5(a)]. These transitions involve states of mainly $d$ character, as quantified at the bottom of panel (b). Interestingly, all the electronic transitions computed $<1 \mathrm{eV}$ (relevant for RF), involve $\beta$ spin states, showing the relevance of the cluster's high spin for the presence of low-lying electronic excitations, which ultimately determines its high RF rate constants. As is shown in Fig. 3(b), the agreement between experiment and theory is outstanding. This nearly perfect agreement may be partly fortuitous, given the expected accuracy of a TD-DFT calculation, although reproducing both the absolute values and the size dependence is remarkable. The accordance provides clear evidence for the assignment of the suppression of the metastable decay to radiation from thermally excited electronic states via the process of RF.

\section{CONCLUSIONS}

In this paper, we have determined the rates of thermal photon emission for $\mathrm{Co}_{n}^{+}(n=5-23)$ clusters, obtaining the highest values ever reported for a metallic cluster system. Moreover, we have shown a quantitative agreement between the measured radiation rates and those modeled with DFT calculations. The success of our calculations, which contrasts to those for other systems like gold clusters, can be traced to the simpler, highly populated, electronic structure of the first-row transition metal cobalt, with excitations that can be fairly well described by single electron transitions. In contrast, for more complicated systems like gold, collective excitations, which are not described by DFT, can play a fundamental role in their radiative cooling mechanism [6] and require further analysis.

\section{ACKNOWLEDGMENTS}

This paper has been supported by the Research Foundation-Flanders (FWO, Projects No. G0A05.19N and No. AKUL/15/16 G0H1516N) and by the KU Leuven Research Council (Project No. C14/18/073). P.F. is a Senior Postdoctoral Fellow of the FWO. The resources and services used for the computations in this paper were provided by the VSC (Flemish Supercomputer Center), funded by the FWO and the Flemish Government. Part of the work has been performed under the Project HPC-EUROPA3 (No. INFRAIA-2016-1-730897), with the support of the EC Research Innovation Action under the H2020 Programme. P.F. gratefully acknowledges the support of the Department of Inorganic and Organic Chemistry at the University of Barcelona and the computer resources and technical support provided by the Barcelona Supercomputer Centre.
[1] R. C. Dunbar, Infrared radiative cooling of isolated polyatomic molecules, J. Chem. Phys. 90, 7369 (1989).
[2] J. Bernard, A. Al-Mogeeth, A.-R. Allouche, L. Chen, G. Montagne, and S. Martin, Photo-dissociation of naphthalene 
dimer cations stored in a compact electrostatic ion storage ring, J. Chem. Phys. 150, 054303 (2019).

[3] P. Ferrari, E. Janssens, P. Lievens, and K. Hansen, Radiative cooling of size-selected gas phase clusters, Int. Rev. Phys. Chem. 38, 405 (2019).

[4] A. Nitzan and J. Jortner, Theory of inverse electronic relaxation, J. Chem. Phys. 71, 3524 (1979).

[5] S. Leach, Photophysics, electronic spectroscopy and relaxation of molecular ions and radicals with special reference to polycyclic aromatic hydrocarbons, in Polycyclic Aromatic Hydrocarbons and Astrophysics (D. Reidel Publishing Company, Dordrecht, Holland, 1987), pp. 99-127.

[6] K. Hansen, P. Ferrari, E. Janssens, and P. Lievens, Thermal radiation of gold clusters on microsecond time scales, Phys. Rev. A 96, 022511 (2017).

[7] K. Hansen and E. E. B. Campbell, Radiative cooling of fullerenes, J. Chem. Phys. 104, 5012 (1996).

[8] S. Martin, J. Bernard, B. Brédy, B. Concina, C. Joblin, M. Ji, C. Ortega, and L. Chen, Fast Radiative Cooling of Anthracene Observed in a Compact Electrostatic Storage Ring, Phys. Rev. Lett. 110, 063003 (2013).

[9] V. Chandrasekaran, A. Prabhakaran, B. Kafle, H. Rubinstein, O. Heber, M. Rappaport, Y. Toker, and D. Zajfman, Formation and stabilization of $\mathrm{C}_{6}^{-}$by radiative electron attachment, J. Chem. Phys. 146, 094302 (2017).

[10] E. A. Rohlfing, Optical emission studies of atomic, molecular, and particulate carbon produced from a laser vaporization cluster source, J. Chem. Phys. 89, 6103 (1988).

[11] U. Frenzel, A. Roggenkamp, and D. Kreisle, 'Black-body' radiation of free niobium clusters heated by oxidation, Chem. Phys. Lett. 240, 109 (1995).

[12] R. Mitzner and E. E. B. Campbell, Optical emission studies of laser desorbed $\mathrm{C}_{60}$, J. Chem. Phys. 103, 2445 (1995).

[13] Y. Ebara, T. Furukawa, J. Matsumoto, H. Tanuma, T. Azuma, H. Shiromaru, and K. Hansen, Detection of Recurrent Fluorescence Photons, Phys. Rev. Lett. 117, 133004 (2016).

[14] M. Saito, H. Kubota, K. Yamasa, K. Suzuki, T. Majima, and H. Tsuchida, Direct measurement of recurrent fluorescence emission from naphthalene ions, Phys. Rev. A 102, 012820 (2020).

[15] F.-Q. Chen, N. Kono, R. Suzuki, T. Furukawa, H. Tanuma, P. Ferrari, T. Azuma, J. Matsumoto, V. Zhaunerchykf, and $\mathrm{K}$. Hansen, Radiative cooling of cationic carbon clusters, $\mathrm{C}_{N}^{+}$, $N=8$, 10, 13-16, Phys. Chem. Chem. Phys. 21, 1587 (2019).

[16] A. E. Green, A. S. Gentleman, W. Schöllkopf, A. Fielicke, and S. R. Mackenzie, Atomic Cluster $\mathrm{Au}_{10}^{+}$Is a Strong Broadband Midinfrared Chromophore, Phys. Rev. Lett. 127, 033002 (2021).

[17] V. Zamudio-Bayer, K. Hirsch, A. Langenberg, A. Ławicki, A. Terasaki, B. von Issendorff, and J. T. Lau, Large orbital magnetic moments of small, free cobalt cluster ions $\mathrm{Co}_{n}^{+}$with $n \leqslant 9$, J. Phys.: Condens. Matter 30, 464002 (2018).

[18] A. Langenberg, K. Hirsch, A. Ławicki, V. Zamudio-Bayer, M. Niemeyer, P. Chmiela, B. Langbehn, A. Terasaki, B. v. Issendorff, and J. T. Lau, Spin and orbital magnetic moments of size-selected iron, cobalt, and nickel clusters, Phys. Rev. B 90, 184420 (2014).

[19] E. M. Cunningham, A. E. Green, G. Meizyte, A. S. Gentleman, P. W. Beardsmore, S. Schaller, K. M. Pollow, K. Saroukh, M. Förstel, and O. Dopfer, Infrared action spectroscopy of nitrous oxide on cationic gold and cobalt clusters, Phys. Chem. Chem. Phys. 23, 329 (2021).

[20] J. Jalink, J. M. Bakker, T. Rasing, and A. Kirilyuk, Channeling vibrational energy to probe the electronic density of states in metal clusters, J. Phys. Chem. Lett. 6, 750 (2015).

[21] R. Gehrke, P. Gruene, A. Fielicke, G. Meijer, and K. Reuter, Nature of Ar bonding to small $\mathrm{Co}_{n}^{+}$clusters and its effect on the structure determination by far-infrared absorption spectroscopy, J. Chem. Phys. 130, 034306 (2009).

[22] P. Ferrari, J. Vanbuel, Y. L. T. Li, E. Janssens, and P. Lievens, The double laser ablation source approach, in Gas Aggregation Synthesis of Nanoparticles (Wiley-VCH, Weinheim, 2017), pp. $59-78$.

[23] V. E. Bondybey, Relaxation and vibrational energy redistribution processes in polyatomic molecules, Annu. Rev. Phys. Chem. 35, 591 (1984).

[24] P. Ferrari, K. Hansen, P. Lievens, and E. Janssens, Stability of small cationic platinum clusters, Phys. Chem. Chem. Phys. 20, 29085 (2018)

[25] P. Ferrari, H. A. Hussein, C. J. Heard, J. Vanbuel, P. Lievens, R. L. Johnston, and E. Janssens, Effect of palladium doping on the stability and fragmentation patterns of cationic gold clusters, Phys. Rev. A 97, 052508 (2018).

[26] N. Veldeman, E. Janssens, K. Hansen, J. De Haeck, R. E. Silverans, and P. Lievens, Stability and dissociation pathways of doped $\mathrm{Au}_{n} X^{+}$clusters $(X=\mathrm{Y}, \mathrm{Er}, \mathrm{Nb})$, Faraday Discuss. 138, 147 (2008).

[27] M. Hedén, H. Hansen, and E. E. B. Campbell, Molecular fusion of $\left(\mathrm{C}_{60}\right)_{N}$ clusters in the gas phase after femtosecond laser irradiation, Phys. Rev. A 71, 055201 (2005).

[28] S. Neukermans, E. Janssens, Z. F. Chen, R. E. Silverans, P. v. R. Schleyer, and P. Lievens, Extremely Stable Metal-Encapsulated $\mathrm{AlPb}_{10}^{+}$and $\mathrm{AlPb}_{12}^{+}$Clusters: Mass-Spectrometric Discovery and Density Functional Theory Study, Phys. Rev. Lett. 92, 163401 (2004).

[29] H. Kühlewind, H. J. Neusser, and E. W. Schlag, Metastable fragment ions in multi-photon time-of-flight mass spectrometry: decay channels of the benzene cation, Int. J. Mass Spectrom. Ion. Phys. 51, 255 (1983).

[30] A. Kiermeier, B. Ernstberger, H. J. Neusser, and E. W. Schlag, Multiphoton mass spectrometry of clusters: dissociation kinetics of the benzene cluster ions, J. Phys. Chem. 92, 3785 (1988).

[31] P. Ferrari, E. Janssens, P. Lievens, and K. Hansen, Thermal radiation and fragmentation pathways of photo-excited silicon clusters, J. Chem. Phys. 143, 224313 (2015).

[32] P. Ferrari, J. Vanbuel, K. Hansen, P. Lievens, E. Janssens, and A. Fielicke, Effect of radiative cooling on the size-dependent stability of small boron clusters, Phys. Rev. A 98, 012501 (2018).

[33] J. van der Tol and E. Janssens, Size-dependent velocity distributions and temperatures of metal clusters in a helium carrier gas, Phys. Rev. A 102, 022806 (2020).

[34] L. Geng, C. Cui, Y. Jia, H. Wu, H. Zhang, B. Yin, Z.-D. Sun, and Z. Luo, Reactivity of cobalt clusters $\mathrm{Co}_{n}^{ \pm / 0}$ with ammonia: $\mathrm{Co}_{3}^{+}$cluster catalysis for $\mathrm{NH}_{3}$ dehydrogenation, J. Phys. Chem. A 124, 5879 (2020).

[35] F. Neese, F. Wennmohs, U. Becker, and C. Riplinger, The ORCA quantum chemistry program package, J. Chem. Phys. 152, 224108 (2020). 
[36] J. N. Harvey, On the accuracy of density functional theory in transition metal chemistry, Annu. Rep. Prog. Chem. Sect. C 102, 203 (2006).

[37] J. M. Bakker, J. Jalink, D. Dieleman, and A. Kirilyuk, Structural determination of neutral $\mathrm{Co}_{n}$ clusters $(n=4-10,13)$ through IR-UV two-color vibrational spectroscopy and DFT calculations, J. Phys.: Condens. Matter 30, 494003 (2018).

[38] K. Mehlig, K. Hansen, M. Hedén, A. Lassesson, A. V. Bulgakov, and E. E. B. Campbell, Energy distributions in multiple photon absorption experiments, J. Chem. Phys. 120, 4281 (2004).

[39] K. Hansen, Statistical Physics of Nanoparticles in the Gas Phase (Springer Science \& Business Media, Dordrecht, 2012).

[40] V. Chernyy, L. Logemann, J. M. Bakker, and A. Kirilyuk, The repopulation of electronic states upon vibrational excita- tion of niobium carbide clusters, J. Chem. Phys. 145, 024313 (2016).

[41] V. Chernyy, R. Logemann, A. Kirilyuk, and J. M. Bakker, Direct IR spectroscopic detection of a low-lying electronic state in a metal carbide cluster, ChemPhysChem 19, 1424 (2018).

[42] See Supplemental Material at http://link.aps.org/supplemental/ 10.1103/PhysRevResearch.3.033225 for (i) calculations of $\mathrm{Co}_{5}^{+}$ employing the $2 S+1=13$ spin state, (ii) electronic spectrum of $\mathrm{Co}_{5}^{+}$up to $4 \mathrm{eV}$, (iii) electronic spectrum of $\mathrm{Co}_{n}^{+}(n=5-10)$ up to $1 \mathrm{eV}$, (iv) isomers of $\mathrm{Co}_{5}^{+}$, and (v) $\mathrm{XYZ}$ coordinates of $\mathrm{Co}_{n}^{+}(n=3-10)$.

[43] D. A. Hales, C. X. Su, L. Lian, and P. B. Armentrout, Collisioninduced dissociation of $\mathrm{Co}_{n}^{+}(n=2-18)$ with Xe: bond energies of cationic and neutral cobalt clusters, dissociation pathways, and structures, J. Chem. Phys. 100, 1049 (1994). 